\title{
Magneto-Transport Characterization of Four-Arm Nanostructure Based on Ferromagnetic (Ga,Mn)As
}

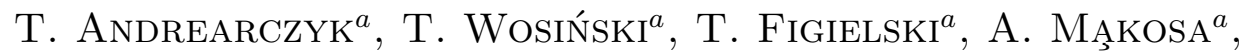 \\ J. SADOWski ${ }^{a, b}$, Z. TKACZYK ${ }^{a}$, E. ŁUSAKOWSKA ${ }^{a}$ \\ AND J. WRÓBEL ${ }^{a}$ \\ ${ }^{a}$ Institute of Physics, Polish Academy of Sciences \\ al. Lotników 32/46, 02-668 Warsaw, Poland \\ ${ }^{b}$ MAX-Lab, Lund University, 22100 Lund, Sweden
}

\begin{abstract}
We report on results of magneto-transport measurements performed on four-arm nanostructure fabricated from $p$-type ferromagnetic $\mathrm{Ga}_{0.92} \mathrm{Mn}_{0.08} \mathrm{As}$ layer. The results reveal hysteresis-like behaviors of low field magnetoresistance. We interpret the magnetoresistance in terms of domain walls, which are expected to be trapped inside the nanostructure at some particular positions and which contribute to the total resistance.
\end{abstract}

PACS numbers: 73.50.Jt, 75.50.Pp, 75.30.Gw, 75.60.Ch

\section{Introduction}

Magneto-transport examination of (Ga,Mn)As ferromagnetic semiconductors is one of the methods of studying their magnetic properties [1]. This method is especially useful for nanostructures, where a relatively small volume of ferromagnetic material makes difficult to measure magnetization in a classical way. The magnetization of ferromagnetic $(\mathrm{Ga}, \mathrm{Mn}) \mathrm{As}$ is known to be governed by the cubic and uniaxial magneto-crystalline anisotropies; cf. [2] and references therein. However, the nanostructurization performed on this material can introduce additional, so-called, patterning (or shape) induced magnetic anisotropy [3, 4]. This fact provides an opportunity to design such a pattern, which distinguishes a few single-domain regions of different anisotropies separated by domain walls (DWs). Spintronics effects related to the charge-carrier transport across individual DWs are the subject of our present research interest. As we recently demonstrated for the case of three-arm (Ga,Mn)As nanostructure [5], we were able to detect DW contribution to the resistance by the magnetoresistance (MR) measurements. Fol- 
lowing this result, we have designed more complex four-arm nanostructures for intended purpose to investigate the fascinating phenomenon of DWs' movement induced by an electric current pulse. Intentionally, one pair of electric terminals in such devices is to be used for DWs' manipulation and the second pair for detection of resulting resistance changes. At the beginning step of this work, we have carried out magneto-transport characterization of different four-arm nanopatterns. In this paper we report on our results concerning DW-dependent MR effects appearing in a simple four-arm nanostructure fabricated from ferromagnetic $\mathrm{Ga}_{0.92} \mathrm{Mn}_{0.08} \mathrm{As}$ layer.

\section{Experimental results}

The $p$-type $\mathrm{Ga}_{0.92} \mathrm{Mn}_{0.08}$ As layer, of the thickness, $d$, of $10 \mathrm{~nm}$, has been grown by low-temperature molecular beam epitaxy (LT-MBE) method on the (001) face of a semi-insulating GaAs substrate. After the growth a thin amorphous As film was deposited on the (Ga,Mn)As surface and the layer was annealed in the $\mathrm{MBE}$ chamber at a temperature of $200^{\circ} \mathrm{C}$ for a duration of $1 \mathrm{~h}$ in order to remove $\mathrm{Mn}$ interstitials from the layer and increase its Curie temperature [6]. Examining the layer by means of a superconducting quantum interference device (SQUID) magnetometer shows the ferromagnetic ordering of Mn ions to occur below $120 \mathrm{~K}$. Hysteresis loops of the layer magnetization have been examined at the liquid helium temperature. The results, shown in Fig. 1, confirm the presence of both the $\langle 100\rangle$ in-plane cubic anisotropy and $\langle 110\rangle$ in-plane uniaxial anisotropy.

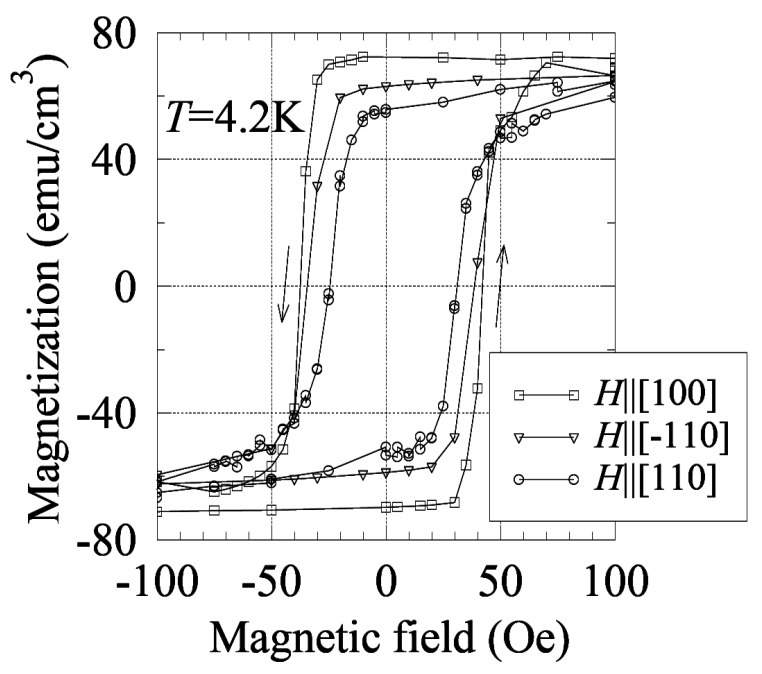

Fig. 1. Hysteresis loop of magnetization as a function of external magnetic field, $H$, for the $\mathrm{Ga}_{0.92} \mathrm{Mn}_{0.08} \mathrm{As}$ layer, measured by the SQUID magnetometer at the temperature $T=4.2 \mathrm{~K}$. The directions of field sweeping are indicated by the arrows. The used configurations of the in-plane magnetic field with respect to the crystallographic directions are given in the legend. 


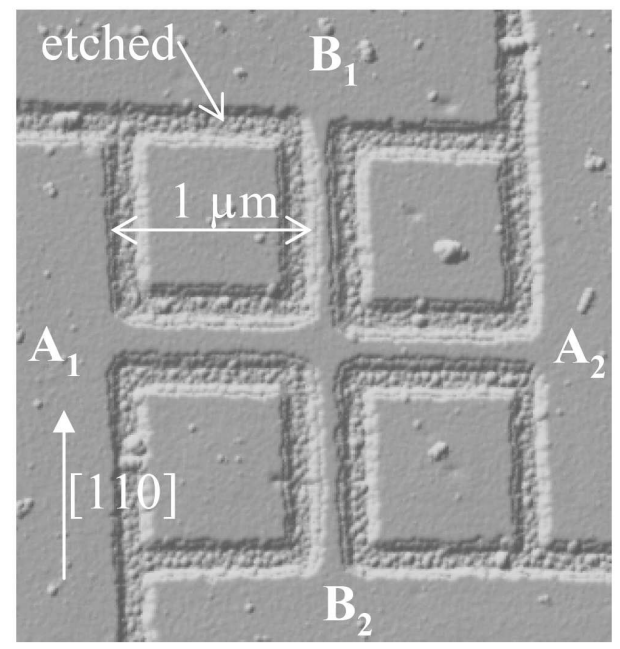

Fig. 2. AFM image of the studied four-arm nanostructure. The area etched to $27 \mathrm{~nm}$ depth is indicated by the arrow. The $\mathrm{A}_{1}, \mathrm{~A}_{2}, \mathrm{~B}_{1}$ and $\mathrm{B}_{2}$ denotations specify the electric terminals to the particular arms (nanowires).

The hysteresis loop obtained for the magnetic field aligned along the [100] easy axis, exhibits a maximum value of the coercive field of $40 \mathrm{Oe}$.

Using electron-beam lithography technique, combined with citric acid etching applied to $\mathrm{Ga}_{0.92} \mathrm{Mn}_{0.08} \mathrm{As}$ layer, we have prepared the four-arm nanostructure, whose atomic force microscopy (AFM) image is shown in Fig. 2. The nanostructure consisted of two perpendicularly crossed wires, oriented along the $\langle 110\rangle$ crystallographic directions, whose width was $100 \div 200 \mathrm{~nm}$, and their length was $2 \mu \mathrm{m}$. Ohmic contacts to the nanowire terminals were prepared by indium soldering.

The charge-carrier transport properties of the four-arm nanostructure were studied at liquid helium temperature when $(\mathrm{Ga}, \mathrm{Mn}) \mathrm{As}$ was in a well-defined ferromagnetic state. Using a low-frequency lock-in technique we measured two-probe resistance as a function of an external magnetic field, in a range comparable to the magnetic coercivity of the parent $(\mathrm{Ga}, \mathrm{Mn})$ As layer. We probed the resistance between either $A_{1}-A_{2}$ or $B_{1}-B_{2}$ pair of terminals (see Fig. 2). For the first pair the zero field resistance is $47 \mathrm{k} \Omega$ while for the second one it is $63 \mathrm{k} \Omega$ at $T=4.2 \mathrm{~K}$. Figure 3 shows the MR of each of the two pairs of terminals, measured for three different configurations of the in-plane external magnetic field. One can distinguish there two different resistive states: the high one, denoted by $R_{\mathrm{H}}$, and the low one, denoted by $R_{\mathrm{L}}$, where $R_{\mathrm{H}}-R_{\mathrm{L}} \approx 100 \Omega$. Let us note that when the high (low) resistance is registered on $\mathrm{A}_{1}-\mathrm{A}_{2}$ terminals, then at the same time the low (high) resistance is registered on $\mathrm{B}_{1}-\mathrm{B}_{2}$ terminals, independently of an actual in-plane field configuration. The in-plane $90^{\circ}$ rotation of the magnetic field direction switches the $R_{\mathrm{L}}\left(R_{\mathrm{H}}\right)$ resistance to $R_{\mathrm{H}}\left(R_{\mathrm{L}}\right)$ one for $\mathrm{A}_{1}-\mathrm{A}_{2}$ pair of terminals and simultaneously the $R_{\mathrm{H}}\left(R_{\mathrm{L}}\right)$ resistance to $R_{\mathrm{L}}\left(R_{\mathrm{H}}\right)$ one for $\mathrm{B}_{1}-\mathrm{B}_{2}$ termi- 


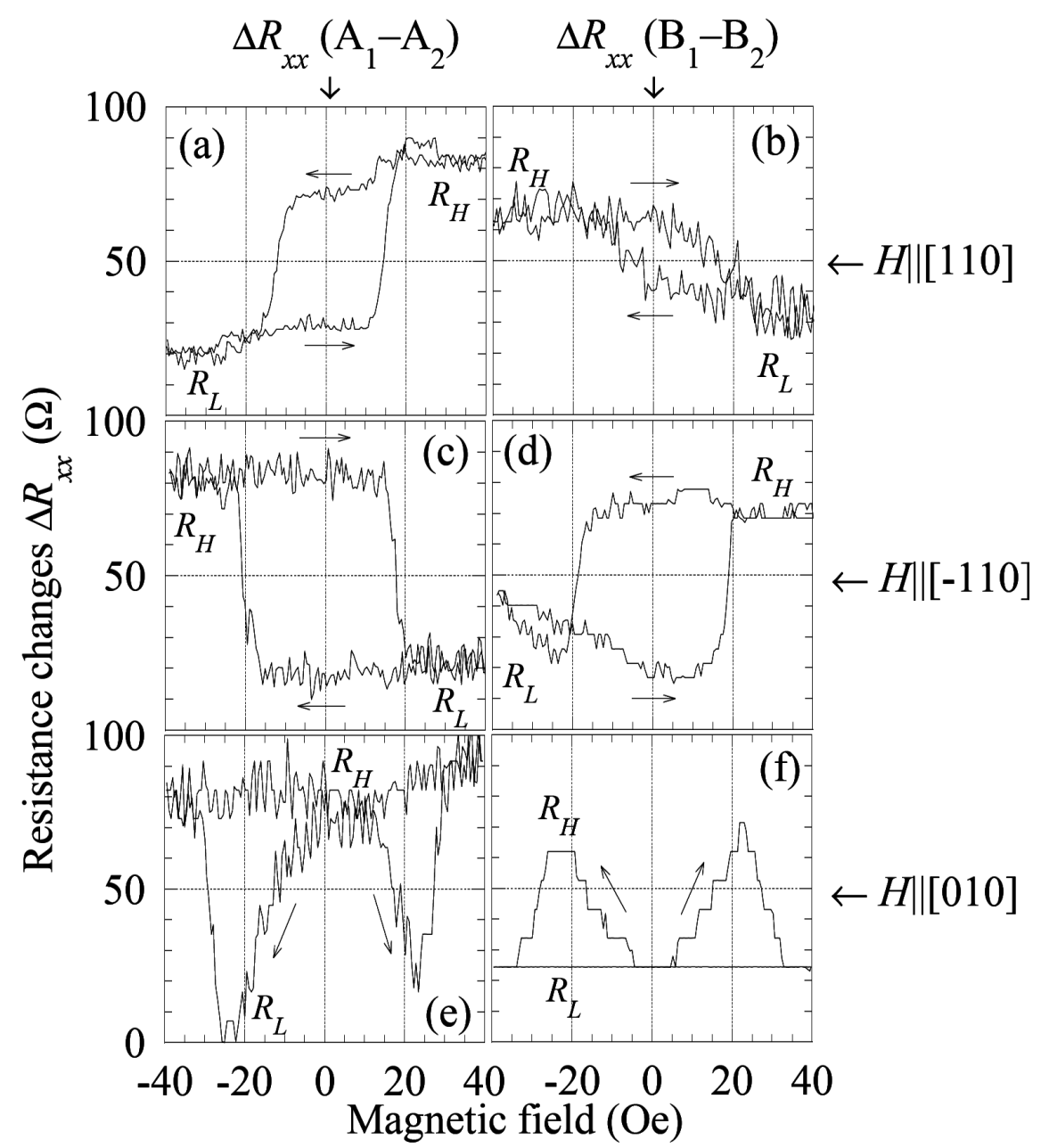

Fig. 3. Resistance changes of the studied four-arm nanostructure in the external in-plane magnetic field at the temperature $T=4.2 \mathrm{~K}$. The two-probe resistance measurements were carried out for two pairs of terminals: for $\mathrm{A}_{1}-\mathrm{A}_{2}((\mathrm{a}),(\mathrm{c})$ and (e)) and for $\mathrm{B}_{1}-\mathrm{B}_{2}((\mathrm{~b})$, (d) and (f)), while the magnetic field was swept in two opposite directions, as indicated by the arrows. The magnetic field configurations with respect to the crystallographic directions are: $H$ parallel to [110] for (a) and (b), $H$ parallel to $[-110]$ for (c) and (d) and $H$ parallel to [010] for (e) and (f).

nals. Moreover, the MR reveals hysteresis-like behavior, when the magnetic field is directed along whatever arms of the nanostructure (i.e., along in-plane $\langle 110\rangle$ crystallographic directions). 


\section{Discussion}

The observed switching between the low and high values of the resistance is here assigned to the magnetic field induced reorientations of the local magnetization vectors inside the nanostructure. The values of the switching field turn out to be less compared to the SQUID coercive fields of the parent layer. This follows likely from a modification of a local magnetic anisotropy due to the nanostructurization. We could expect that, as a consequence of the patterning-induced magnetic anisotropy [3, 4], the in-arm magnetization vectors were directed along the arms' axes, i.e. nearly along $\langle 110\rangle$ crystallographic directions. In reality, due to limited precision of the lithography process, there is some small tilt angle $\left(\delta \approx \pm 2^{\circ}\right)$ between the $\langle 110\rangle$ directions and the arms' axes. Anyway, a relatively low magnetic field is assumed to change the sense of the in-arm magnetization vectors, remaining unchanged their directions.

Importantly, the patterning anisotropy does not apply to the central part of the nanostructure, i.e. to the region where the nanowires cross. We expect that in this region the external magnetic field can switch the local magnetization vector between [100] and [010] easy axes, similarly as it does in the parent layer. That expectation implies the presence of four DWs, separating the central-part single magnetic domain from the in-arm single domains. Consequently, there are two possible tilt angles, $45^{\circ}-\delta$ or $45^{\circ}+\delta$, between the directions of the magnetic moment in the central part of the nanostructure and in any of its arms. After detailed analysis of the all possible switching events for such defined local domains, we assign the low resistive state $\left(R_{\mathrm{L}}\right)$ to the situation, where the two DWs with a magnetic tilt angle $45^{\circ}-\delta\left(\left(45^{\circ}-\delta\right)\right.$ DWs $)$ stand on the way of electric current. Instead, the high resistive state $\left(R_{\mathrm{H}}\right)$ appears when these walls are the two $\left(45^{\circ}+\delta\right)$ DWs. Hence, one can evaluate the contribution of the domain wall to the resistance: $R_{\left(45^{\circ}+\delta\right) \mathrm{DW}}-R_{\left(45^{\circ}-\delta\right) \mathrm{DW}}=(1 / 2)\left(R_{\mathrm{H}}-\mathrm{R}_{\mathrm{L}}\right) d W \approx 0.08 \Omega \mu \mathrm{m}^{2}$, where the average width of the wire, $W=150 \mathrm{~nm}$, has been taken into account. Due to the small $\delta$ angle, the above value is significantly smaller than the value of $12 \Omega \mu \mathrm{m}^{2}$ determined previously for the $R_{136^{\circ} \mathrm{DW}}-R_{44^{\circ} \mathrm{DW}}$ resistance from $\mathrm{MR}$ analysis of a three-arm nanostructure [5].

\section{Conclusions}

The low-field MR measurements were carried out for the four-arm nanostructures fabricated from $p$-type ferromagnetic $\mathrm{Ga}_{0.92} \mathrm{Mn}_{0.08} \mathrm{As}$ layer. The $\mathrm{MR}$ exhibits switching effects between the two well-defined resistive states, including the hysteresis-like behavior. We interpret the MR results taking into account the DWs, which are trapped in the central part of the nanostructure and which contribute to the total resistance in two different ways, depending on the external magnetic field. The DW contribution to the resistance of $0.08 \Omega \mu \mathrm{m}^{2}$ has been deduced for our nanostructure. As the observed switching effects of MR seem to be governed by the $\delta$ angle, we expect in the future to significantly enhance the effects 
by a rotation of the nanostructure pattern with respect to the crystallographic directions in a controlled way.

\section{Acknowledgments}

The work was supported by the Polish Ministry of Science and Higher Education under grant No. N507 461833.

\section{References}

[1] H.X. Tang, R.K. Kawakami, D.D. Awschalom, M.L. Roukes, Phys. Rev. Lett. 90, 107201 (2003).

[2] M. Sawicki, J. Magn. Magn. Mater. 300, 1 (2006).

[3] S. Hümpfner, K. Pappert, J. Wenisch, K. Brunner, C. Gould, G. Schmidt, L.W. Molenkamp, M. Sawicki, T. Dietl, Appl. Phys. Lett. 90, 102102 (2007).

[4] T. Yoo, D. Shin, J. Kim, H. Kim, S. Lee, X. Liu, J.K. Furdyna, J. Appl. Phys. 103, $07 \mathrm{D} 119$ (2008).

[5] T. Figielski, T. Wosiński, A. Morawski, A. Mạkosa, J. Wróbel, J. Sadowski, Appl. Phys. Lett. 90, 052108 (2007).

[6] M. Adell, L. Ilver, J. Kanski, V. Stanciu, P. Svedlindh, J. Sadowski, J.Z. Domagała, F. Terki, C. Hernandez, S. Charar, Appl. Phys. Lett. 86, 112501 (2005). 